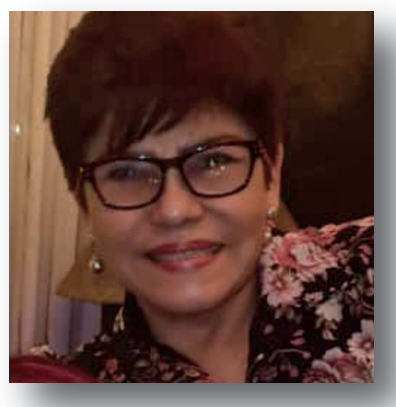

\section{"LA INSTITUCIONALIZACIÓN \\ DEL SISTEMA DE \\ ASEGURAMIENTO DE CALIDAD \\ EN LA EDUCACIÓN SUPERIOR \\ EN HONDURAS"}

\author{
PhD. IVY LOU GREEN ARRECHAVALA \\ ivy.green@unah.edu.hn \\ Universidad Nacional Autonoma de Honduras \\ UNAH \\ ORCID: 0000-0001-8579-4599
}

DOI: $10.5377 /$ rpdd.v5i1.11967

Recibido: septiembre, 2018

Aceptado: noviembre, 2018

\title{
RESUMEN
}

L

a Universidad, de acuerdo con sus fines misionales, es la primera institución sobre la que recae la responsabilidad de la gestión del conocimiento, de la producción científica y transferencia tecnológica para responder a las exigencias de las sociedades modernas (IIPE-UNESCO, 2011).

El presente artículo comunica los resultados y hallazgos principales de la tesis doctoral Institucionalización del Sistema de Aseguramiento de Calidad en la Educación Superior de Honduras, como un aporte a la gestión del desarrollo humano sostenible desde una Educación Superior de Calidad, mostrando desde una perspectiva socioeducativa la dinámica compleja de la gestión de la calidad de la educación superior. A partir de las consideraciones teóricas expuestas se construye una propuesta de análisis de la interrelación entre Educación Superior, Calidad y Desarrollo Humano Sostenible estableciendo un círculo virtuoso que sustenta la institucionalización de un sistema de aseguramiento de la calidad de la educación.

El propósito de la investigación es valorar la viabilidad y la pertinencia de la institucionalización de un Sistema de Aseguramiento de Calidad (SAC) de la Educación Superior de Honduras contribuyendo al Desarrollo Humano Sostenible del mismo. 
Los resultados revelan un avance desigual en cuanto a los estadios de madurez de la institucionalización del sistema de aseguramiento de calidad de la educación superior del país, mostrando la inexistencia de un imaginario común en torno a la calidad de la educación superior, su gestión y su aporte al Desarrollo Humano Sostenible (DHS), develando la necesidad de desarrollar estrategias de implantación e implementación del Sistema de Aseguramiento de Calidad para el aseguramiento de la calidad de la educación superior del país potenciándola como eje fundamental para el DHS.

\title{
PALABRAS CLAVES
}

Educación Superior, Calidad, Desarrollo Humano Sostenible, Aseguramiento de Calidad.

\begin{abstract}
The University, according to its missionary aims, is the first institution on which the responsibility of knowledge management, scientific production and technology transfer falls to respond to the demands of modern societies. (IIEP-UNESCO, 2011).

This article communicates the results and the main findings of the doctoral thesis Institutionalization of the Quality Assurance System in Higher Education of Honduras, as a contribution to the management of Sustainable Human Development from a Higher Education of Quality, showing from a socio-educational perspective the complex dynamics of the management of the quality of higher education. Based on the theoretical considerations presented, a proposal is made to analyse the interrelationship between Higher Education, Quality and Sustainable Human Development, establishing a virtuous circle that supports the institutionalization of a quality assurance system for education.
\end{abstract}

The purpose of the research is to assess the feasibility and relevance of the institutionalization of a Higher Education Quality Assurance System in Honduras contributing to its Sustainable Human Development.

The results reveal an uneven progress in terms of the stages of maturity of the institutionalization of the quality assurance system of higher education in the country, showing the inexistence of a common imaginary regarding the quality of higher education, its management and its contribution to Sustainable Human Development, revealing the need to develop strategies for the implementation of the Quality Assurance System for quality assurance of higher education in the country, strengthening it as a fundamental axis for Sustainable Human Development. 


\section{KEYWORDS}

Higher Education, Quality, Sustainable Human Development, Institutionalization, Quality Assurance

\section{INTRODUCCIÓN}

Este artículo presenta de manera resumida la tesis doctoral acerca de la Institucionalización de un Sistema de Aseguramiento de Calidad de la Educación Superior como una contribución a la comunidad académica en conocimientos para el establecimiento de mecanismos para mejorar la calidad de la educación y el Desarrollo Humano de la población hondureña.

La investigación se justifica en la necesidad de atender la problemática de calidad existente de la educación superior hondureña, la cual se caracteriza por altos niveles de exclusión de la población, baja producción científica, insatisfacción de los graduados, poco aporte a la solución de los problemas de la sociedad y un proceso de internacionalización poco desarrollado.

Los altos índices de pobreza y de desigualdad social que impera en el país, es la razón ineludible que obliga a repensar el tema de la calida d de la educación superior en términos del desarrollo de Honduras, promoviendo estrategias que permitan establecer una educación más pertinente y equitativa, que contribuya con la superación de tales asimetrías en el desarrollo humano de la población hondu reña.

La actualidad de la tesis se sustenta en el interés cada vez más creciente por la mejora de la calidad de la educación superior tal como lo evidencian los grandes relatos universales sobre educación superior, promovidos por UNESCO y sus Conferencias Mundiales, los Objetivos de Desarrollo del Milenio (ODM), la Educación para Todos (EPT) la Agenda 2030 para el Desarrollo Humano Sostenible y las iniciativas de mejora de calidad, innovación y armonización del CSUCA, con sus Planes de Integración Regiona 1. PIRESC II.

Se deja establecida la relevancia del tema de investigación en tanto viene a producir datos que hasta el momento no se tienen. Se conocen datos de la región, existen estudios internacionales pero no se cuenta con información científica propi a del país sobre este tema. Es un estudio necesario porque le aporta al país nuevos conocimientos sobre una de sus áreas críticas que debería estar vinculada con todos los niveles educativos del país: la Educación Superior y su calidad. 
Finalmente, el estudio es pertinente, ya que en el país el Sistema de Educación Superior ha crecido pero no existen evidencias científicas acerca de su calidad. Es coherente, en tanto es un tema de actualidad, se evalúan las empresas, la economía, se evalúa el nivel básico la educación media y sus docentes, pero qué se ha dicho de la educación superior . ¿Cómo no va ser necesario investigar las organizaciones sobre quienes recae la responsabilidad de la producción científica, tecnológica, la formación de los profesionales q ue son el futuro del país?.

Se sostiene en la tesis que para mejorar la contribución de la Educación Superior al Desarrollo Humano Sostenible es necesario que las instituciones de Educación Superior revis en sus esquemas de gestión y se enfoquen en esquemas de evaluación y acreditación, de calidad con enfoque hacia el desarrollo humano sostenible.

Desde tal perspectiva, es necesario el estudio de los requerimientos y el sorteo de posibles obstáculos que enfrenta la institucionalización de un sistema de aseguramiento como herramienta para la mejora de la calidad y pertinencia de la educación superior en Honduras. Por ello, una mirada reflexiva crítica desde la teoría de la gestión de la calidad, la gestión del cambio y la mejora de las instituciones que aprenden constituye una novedad y posibilita una toma de conciencia de lo omitido.

\section{METODOLOGÍA}

La investigación es de tipo cualitativo. Las técnicas que se aplicaron son: revisión documental, observación participante, entrevista en profundidad y grupo focal.Se realiza una aproximación fenomenológica al significado que otorgan los actores claves de la gestión universitaria, a los procesos de institucionalización de un sistema de aseguramiento de calidad (SAC) en el contexto del Sistema de Educación Superior de Honduras.

En la misma participaron: altas autoridades universitarias del Sistema de Educación Superior de Honduras como gestores de la educación superior, responsables de asegurar la entrega de una educación de calidad a la sociedad hondureña y representantes del imaginario colectivo y de la visión institucional, académicos responsables de unidades de evaluación y acreditación de calidad en las instituciones de educación superior de Honduras y académicos centroamericanos que tienen la experiencia en temas de gestión de calidad y que tienen muchos años participando en los procesos regionales de desarrollo de capacidad para la gestión de calidad y la mejora educación superior. 


\section{LA INSTITUCIONALIZACIÓN DEL SISTEMA DE ASEGURAMIENTO DE CALIDAD EN LA EDUCACIÓN SUPERIOREN HONDURAS}

Para el análisis de los datos, siguiendo el desarrollo del método fenomenológico se llegó a la construcción de una estructura mostrativa de las concepciones y significados de las vivencias de los académicos en relación con el proceso de institucionalización de un sistema de aseguramiento de calidad de la educación superior al servicio del desarrollo humano sostenible de nuestro país.

\section{Pilares conceptuales de la investigación Desarrollo humano Sostenible}

El aparato conceptual crítico que sustenta el estudio se construye en primer lugar a partir de los postulados del desarrollo Humano Sostenible.

Entre las teorías consideradas, destaca la concepción de las libertades, capacidades y necesidades fundamentales para el bienestar de las personas desde los aportes de Max Neff (1993) en el tema de satisfacción de necesidades y Max Neff (2000) ampliando su teoría de las necesidades con el de libertades; Sen (1997) con la propuesta de ampliación de capacidades humanas y Sen, A ( con la discusión acerca de las condiciones mínimas necesarias; Rawls ( que introduce el concepto de justi cia como equidad ; Sen y Nussbaum,1996 con el tema de las “opciones, para mejorar su calidad de vida" y Serrano (2015) con su propuesta del Desarrollo Humano Sostenible como la existencia de condiciones y relaciones humanas necesarias.

Se toma como marco de referencia operativo los Pilares del desarrollo humano propuesta de PNUD: equidad, sostenibilidad, crecimiento económico, participación ciudadana y seguridad humana.

Los postulados descritos sobre el DHS dejan ver claramente que las necesidades humanas van más allá de lo material, abarca al menos cinco dimensiones: social, político, económico, ético y cultural, en conjunto con las interpretaciones económicas, biológicas y ambientales, las cuales son fundamentales para explicar y justificar el desarrollo de un grupo, sociedad o país.

Por lo tanto en esta tesis se considera que desarrollo es un término que debe ser utilizado con mayor propiedad, es un concepto que trata de integrar las dimensiones humanas, sociales, ambientales, éticas, políticas, cultura les y económicas de las condiciones de vida de las personas a través del concepto de "desarrollo integral".

\section{Educación para el desarrollo Humano}

El segundo pilar conceptual que constituye el aparato crítico de la tesis es el de educación para el Desarroll o Humano Sostenible vinculado al concepto de educación 
de calidad y sustentado en que el desarrollo de una nación implica la ampliación de las opciones de la gente, que se logra cuando se aumentan las capacidades y los funcionamientos valiosos de las personas en la vida" (PNUD,2015)(PNUD,2015).

La educación puede contribuir al desarrollo, por un lado, en la medida que se convierte en un "proceso que aumenta la libertad efectiva de las personas para llevar a cabo aquello que valoran" y por otro el pleno ejerc icio del derecho a la educación. Difícilmente se podrá acceder a un empleo digno, o ejercer la libertad de expresión o de participación, si no se tiene buena educación, que ha fortalecido las competencias necesarias que permitan asegurar la inserción de las persona s a la vida sociocultural y productiva global vinculado al desarrollo humano sostenible del país.

Con los aportes de Mesa (2000 y Boni (2005) se presenta una aproximación histórico estructural sobre el desarrollo y madurez de la educación para el desarrollo el nuevo paradigma de la Educación para el DHS); se distinguen cinco "generaciones" o momentos en la evolución de la educación para el desarrollo tomando en cuenta, en primer lugar, los factores externos como: el contexto y problemática internacional de cada periodo; las concepciones predominantes sobre el desarrollo, el subdesarrollo y otros problemas globales conexos, y los actores y fuerzas sociales más importantes en ese contexto.

En cuanto a Educación para el desarrollo Caballero (2004), propone que la educación debe "Favorecer el desarrollo humano sostenible en los tres niveles que afectan a las personas: individual, comunitario-local e internacional"; y por su parte Boni (2005) desaarrolla su propuesta de la Educación para la ciudadanía global; Argibay Celorio (2005) en consideración a la Educación para el Desarrollo (ED), apuntan el gran reto es construir una educación para ser y para transformar. Para las autoras mencionadas es necesario fundamentar la ED desde teorías educativas transformacionales que se centran en la persona, tales como: la Educación humanista (Habermas); Educación popular liberadora (Paulo Freire); Pedagogía crítica (Henry Giroux, Michael Apple, Peter McLaren); el constructivismo (Piaget, Vigotsky, Luria, Ausubel, Novak, Bruner); Investigación -acción participativa (Lewin, Carr, Kemmis) y Pensamiento complejo (Edgar Morin).

Se agrega al tema las demandas a la educación superior en atención a las nuevas dinámicas del contexto (UNESCO, 2009); Van Ginkel (1998), Tunerman (2007), Dridikson (2000) en el debate del rol de la Educación Superior en la Agenda del DHS; la agenda 2030 (PNUD, 2015); Tunerman (2007), Boni y Gasper,(2011): las políticas para el desarrollo y transformación de la educación superior y Gazzola y Didriksson (2008) sobre la Responsabilidad social. 


\section{LA INSTITUCIONALIZACIÓN DEL SISTEMA DE ASEGURAMIENTO DE CALIDAD EN LA EDUCACIÓN SUPERIOREN HONDURAS}

\section{Calidad de la educación}

Otro aspecto de este segundo pilar conceptual es el de calidad de la educación y su gestión. De Yarzábal (2002) y la declaración de Incheón (2015) se establece la relación Educación, Calidad y DHS. Gonzales y Espinoza (2008), Arrien citado por Tunerman (2008), Schmelkes (1994) y Latapi (2009) proponen posturas teóricas con respecto al concepto de calidad de la educación. Siguiendo a Lujambio (2010) se establece los principios de la calidad de la educación: equidad, pertinencia, relevancia, eficacia y eficiencia. Lujambio (2010), Booth y Ainscow (2000) y Tomasevski (2001, 2005) aportan al debate acerca de la equidad. Desde la concepción de responsabilidad social se agrega como principio de calidad el respeto a los derechos humanos.

Seibold (2000) aporta a la discusión de tres factores que inciden en la conformación de la calidad en una institución educativa: el sociocultural, el institucional-organizativo y el didáctico-pedagógico: Estos factores se reconfiguran desde la propuesta de UNESCO (2005) en cinco factores que son:

-Características de los educandos: basada en la idea de que "los educandos no llegan a las aulas en condiciones de igualdad."

-Contexto: La educación debería reflejar fielmente los valores y actitudes de las sociedades. Aportar al desarrollo humano sostenible.

-Aportes materiales y humanos: Esta categoría engloba los recursos materiales y los recursos humanos

-Enseñanza y aprendizaje: Este aspecto está relacionado con lo que ocurre en las aulas y en la escuela

-Resultados: Esta dimensión de la educación se puede expresar en términos de aprovechamiento escolar y de adquisición de ventajas sociales y económicas de carácter más general.

Bravlasky (2004) por su lado identifica diez factores determinantes para una calidad de educación para todos. Estos factores son: el foco en la pertinencia personal y social; la convicción, la estima y la autoestima de los involucrados; la fortaleza ética y profesional de los maestros y profesores; la capacidad de conducción de los directores e inspectores; el trabajo en equipo dentro de la escuela y los sistemas educativos; las alianzas entre las escuelas y los otros agentes educativos; el currículo en todos sus niveles; la cantidad, calidad y disponibilidad de materiales educativos; la pluralidad y calidad de las didácticas; y los mínimos materiales y los incentivos socioeconómicos y culturales.(págs. 22-36)

Se muestra el concepto de calidad como un concepto multidimensional y multirreferencial que responde a elementos históricos y contextuales. Así mismo la 
gestión de calidad requiere de una operacionalización del concepto. Existen muchos modelos de gestión de Calidad y desde las propuestas de Rico (2011), Gonzales y Espinoza (2008) y Lepeley (2011) se discute el tema de la gestión de calidad de la educación. Se presentan diferentes modelos de gestión que han surgido en las empresas tales como el Modelo Deming (Japón), el Modelo Baldrige (Estados Unidos), el EFQM (Europeo) los cuales han sido adaptados a la gestión de la educación superior. Se completa la construcción teórica con la conceptualización de los Sistemas de Aseguramiento de Calidad.

\section{Institucionalización de Sistemas de Aseguramiento de Calidad}

El tercer concepto de base de esta investigación es el de la institucionalización de sistemas de aseguramiento de calidad. Se discuten los conceptos desde las propuestas de Bernal, Martínez, Parra, \& Jiménez (2015), Lemaitre y Zenteno (2012), Espinoza y Gonzáles (2012), Espinoza (2010), De Miguel y Apodaca, (2009) y la estrategia operativa de institucionalización de organizaciones propuestos por Tolbert y Zucker (1996), Silimpert (2012) y operacionalizado por Contreras (2011) y Ruiz, Contreras y Gutiérrez (2013).

Los principales resultados de la investigación de acuerdo con los objetivos planteados son:

\section{1.-Nivel de avance en el proceso de institucionalización del SAC}

El sistema de aseguramiento de calidad en las instituciones de Educación Superior de Honduras tienen un desarrollo bastante desigual, en la poca experiencia desarrollada en el ámbito nacional prevalecen los mecanismos orientados en los propósitos de control de calidad y de garantía de calidad, en forma incipiente. El control de calidad, es un mecanismo obligatorio, el licenciamiento está a cargo del Consejo de Educación Superior y su órgano operativo, la Dirección de Educación Superior ${ }^{1}$. No obstante, este procedimiento no está visto de manera integral, ni está totalmente normado para poder ser considerado un mecanismo de aseguramiento de calidad.

${ }^{1}$ Ley de Educación Superior, las normas académicas y el artículo 160 de la constitución de la república 


\section{LA INSTITUCIONALIZACIÓN DEL SISTEMA DE ASEGURAMIENTO DE CALIDAD EN LA EDUCACIÓN SUPERIOREN HONDURAS}

Uno de los primeros signos de un proceso de institucionalización de un sistema de aseguramiento de calidad en su etapa de pre-institucionalización, es el manejo de una concepción de calidad y la forma de gestionarla estableciendo algún tipo de organización o estructura. La concepción de calidad que prima entre los directivos entrevistados se enmarca en las dimensiones de pertinencia, eficacia y eficiencia. Se pone al descubierto dos omisiones importantes: equidad y relevancia.

Por otro lado, con excepción de una de las autoridades entrevistadas, en la narrativa de los entrevistados hacen falta expresiones que muestre compromiso con temas inherentes inherentes a la calidad y al DHS: responsabilidad social; acceso, equidad y calidad, ética, seguridad humana, participación ciudadana, derechos humanos; internacionalización; regionalización, globalización y aprendizaje, investigación e innovación. Los entrevistados se aproximaron básicamente a dos de las categorías conceptuales mencionadas, lo que se interpreta en la inexistencia de un imaginario común en torno a la calidad de la educación superior y su aporte al DHS.

Entre las experiencias se observa que las universidades públicas han desarrollado esencialmente procesos con fines de mejora, en tanto las universidades privadas se orientan más hacia los mecanismos de garantía de calidad. En general la mayoría de las instituciones que han desarrollado acciones de mejora, como consecuencia de autoevaluaciones, evaluaciones o auditorias, no siempre han estado articuladas a un sistema interno de gestión de calidad.

En los pocos casos donde existen estructuras encargadas de promover la gestión de calidad, no se perciben acciones dentro de una visión estratégicas y prospectiva de calidad. Estas no funcionan de manera articulada y sistemática, porque hace falta implantar sistemas internos de aseguramiento de calidad de la educación.

\section{2.-Obstáculos y desafíos para lograr la institucionalización de un sistema de Aseguramiento de Calidad.}

A nivel interno de las instituciones, se identifica los siguientes obstáculos:

- Uno de los problemas más críticos es la falta de profesionales formados en procesos de aseguramiento de calidad, en temas de evaluación y gestión de calidad.

- Existencia de grupos que se resisten a asumir responsabilidades para la gestión de calidad

- Actitudes de desconfianza indiferencia y conformismo de las autoridades superiores y mandos intermedios.

- Carencia de políticas institucionales de calidad.

- Amenaza de un sistema que tenga injerencia política en la toma de decisiones; la 
influencia planificada y continua de grupos o cuotas de poder económico que miran la educación superior como un bien mercantil tradicional sin necesidad de reformas, cambios o mejoras.

- Existencia de un modelo de acreditación que no sea pertinente o con un compromiso débil ante sus funciones.

- Avances discursivos mas no en la toma de decisiones para la puesta en marcha de acciones tendientes al fortalecimiento de la gestión de calidad en las instituciones.

Como desafíos a nivel interno de las instituciones se identifican:

- Establecer viabilidad administrativa-financiera, técnica, política y tecnológica comunicativa.

- Sensibilizar a los actores universitarios (autoridades, profesores, administrativos y estudiantes) acerca de las áreas críticas que requieren someterse a procesos de mejora de la calidad, la organización de estructuras responsables de la gestión institucional y académica, la formación de cuadros técnicos encargados del aseguramiento de calidad, los apoyos tecnológicos necesarios para lograr mayor eficiencia en los procesos de gestión.

- La falta de presupuesto en las instituciones para evaluación y mejora

-Producto del grupo focal se identificaron como desafíos: la articulación del aseguramiento de calidad al sistema de planificación institucional, falta de sensibilidad de la comunidad universitaria frente a los temas de aseguramiento de calidad, la asunción de la calidad como compromiso de todos y todas los que integran la organización, incipientes capacidades en gestión y calidad.

\section{3.-Condiciones que favorecen y hacen posible la institucionalización del Sistema de Aseguramiento de la Calidad de la Educación Superior}

La valoración de la viabilidad y pertinencia del Sistema de Aseguramiento de Calidad por los actores claves del sistema de Educación Superior es que actualmente tiene creciente legitimación social en Honduras a través del Plan Estratégico de Desarrollo de la Educación Superior (2014) y la creación del SHACES en el 2010. La importancia del SHACES ha llevado a que las 20 universidades hondureñas, que son miembros del Consejo Técnico Consultivo de la Educación Superior participen en la discusión y ejecución de planes y acciones para la puesta en marcha del SHACES.

En el ámbito externo, el Sistema Hondureño de Acreditación de la Educación Superior se ha convertido en el esfuerzo inicial, de la conjunción de varias voluntades para institucionalizar un sistema de aseguramiento de la calidad educativa de Honduras. 


\section{LA INSTITUCIONALIZACIÓN DEL SISTEMA DE ASEGURAMIENTO DE CALIDAD EN LA EDUCACIÓN SUPERIOREN HONDURAS}

Contar con una normativa nacional (SHACES) producto del consenso de todas las universidades que integran el sistema de educación superior viabiliza la instauración de sistemas tanto internos como externos de aseguramiento de calidad.

El respaldo de organismos de alta relevancia como el CSUCA, organismo que promueve la cultura de calidad en las universidades centroamericanas y del CCA, sistema de acreditación de calidad y de promoción de la calidad a nivel de agencias acreditadoras e instituciones educativas, activan energías para trabajar iniciativas encaminadas en la institucionalización del aseguramiento de calidad de la educación superior.

\section{4.-Aporte de una Educación Superior de Calidad en favor del desarrollo humano sostenible en Honduras: Pertinencia del sistema de Aseguramiento de Calidad}

Todavía las agendas educativas no incluyen una visión hacia el Desarrollo Humano Sostenible y no se basa en una Educación para el Desarrollo humano Sostenible. Estos son temas que aún no se reflexionan de manera sistemática en nuestro contexto, en nuestros círculos académicos e institucionales, salvo como iniciativas aisladas y poco articuladas en el curriculum, o como excepción en una institución se declara y desarrolla en el modelo educativo pero aún falta recorrido para llegar a la concreción en el aula y en los aprendizajes.

5.-Las principales responsabilidades institucionales y tareas a ejecutar para la institucionalización del Sistema de Aseguramiento de la calidad de la Educación Superior en Honduras.

Se propone un Plan para la Institucionalización de un Sistema de Aseguramiento de calidad de la Educación Superior en Honduras, cuyo ámbito de incidencia trasciende toda la estructura del sistema universitario en función de los requerimientos del ser humano, la sociedad y la naturaleza y de las funciones sustantivas de las IES.

En el ámbito interno, un Sistema Interno de Gestión de Calidad contempla el quehacer administrativo y el quehacer académico de la institución. El quehacer administrativo responsable de la gestión institucional y el quehacer académico responsable de la gestión de la docencia, investigación, vinculación universidad sociedad (proyección social) y de la internacionalización de la educación. Ambos deben desarrollar un ejercicio de autorregulación.

En el ámbito externo el Sistema de Aseguramiento de calidad se concreta, por un lado, en los vínculos, acciones y logros entre el SHACES y las IES. Dichas acciones pueden ser de control, de garantía y de fomento de la mejora de la calidad 
de las Instituciones de Educación Superior, respaldada institucionalmente por el SHACES (organismo autónomo creado para tal fin a instancias de las instituciones que integran el sistema de educación superior). Por otro lado, también se concreta en las incidencias que desde las IES se configuran en un ejercicio de corresponsabilidad con los distintos sectores de la sociedad local, nacional y global desde la docencia, la investigación, vinculación universidad sociedad e internacionalización.

\section{CONCLUSIONES}

En cuanto a los niveles de avance en la implementación dela Institucionalización del Sistema de Aseguramiento de Calidad los signos del contexto hondureño revelan que el proceso se encuentra entre la fase de habituación y la de objetivación. Es característica de la fase de habituación, la existencia de conciencia de la necesidad de mejora de la calidad de la educación, y en la fase de objetivación, estructuras responsables y pautas de comportamiento en relación con la gestión de calidad.

En el ámbito externo, el SHACES se convierte en el esfuerzo conjunto de varias voluntades para institucionalizar un sistema de aseguramiento de la calidad educativa. Cuenta con una normativa nacional (SHACES) producto del consenso de todas las universidades que integran el sistema de educación superior, el respaldo de organismos de alta relevancia como el CSUCA, el CCA y relaciones con otras agencias e instituciones homólogas.

La institucionalización de un Sistema de Aseguramiento de Calidad de Educación Superior para el Desarrollo Humano Sostenible en Honduras, se viabiliza gracias a las sinergias que existen en el Sistema de Educación Superior y el Consejo Centroamericano de Acreditación, sin embargo es necesario que se desarrollen acciones concretas de acreditación de calidad, de implementación de Sistemas Internos de Gestión de Calidad que garanticen la calidad de los procesos, productos y resultados así como la gestión de mejora permanente y sistemática.

La tesis de esta tesis es compartida dentro del imaginario de las autoridades universitarias, la institucionalización del sistema de aseguramiento de calidad orientará de manera más precisa los grandes fines institucionales y las estrategias necesarias para lograrlas, estableciendo claramente los criterios e indicadores de impacto en el desarrollo humano sostenible de Honduras. Es decir, desarrollar una educación superior orientada a aportar, desde sus funciones sustantivas, a la satisfacción de necesidades o demandas públicas de la población del país, de la región, colocando en el centro la problemática al ser humano y su bienestar, los desafíos de desarrollo sostenible y transformación de las sociedades. Poco o nada aportará la educación 


\section{LA INSTITUCIONALIZACIÓN DEL SISTEMA DE ASEGURAMIENTO DE CALIDAD EN LA EDUCACIÓN SUPERIOREN HONDURAS}

superior hondureña si no se enfoca en esquemas de evaluación y acreditación de calidad que incluya estándares que aseguren el DHS.

Para ello se debe atender con urgencia la definición y puesta en marcha de un plan estratégico y prospectivo, la definición y adopción de políticas de aseguramiento de calidad con su normativa, estrategias, organización y presupuesto, la formación y empoderamiento profesional en gestión de calidad de la educación, el desarrollo de capacidades institucionales para la gestión de calidad y la planificación prospectiva del aseguramiento de calidad y de rendición de cuentas de la educación superior de Honduras.

\section{BIBLIOGRAFÍA}

Aguerrondo I. (1998). América Latina y el Desafío del Tercer Milenio. Educación de Mejor Calidad con Menores Costos. PREAL (Programa de promoción de la Reforma Educativa en América Latina y el Caribe). Enero $\mathrm{N}^{\circ} 10$. Recuperado de http://www. thedialogue.orgwww.thedialogue.org/PublicationFiles/PREAL\%2010-Spanish.pdf

Argibay, M. y Celorio, G. (2005). La educación para el desarrollo. País Vasco: VitoriaGasteiz, Servicio Central de Publicaciones del Gobierno Vasco.

Alarcón A, F., (2012) El CSUCA, en la educación para la integración. Quinta mesa redonda Educación para la integración. Recuperado de http://www. derechocomunitario.ucr.ac.cr/index.php?view $=$ article \&catid=28\%3A1\&id=121\%3 Acsuca-educacion-integracion-alarcon-alba\&format $=$ pdf\&option $=$ com_content

Bernal, S.D; Martínez, M.L; Parra, A. y Jiménez, J.L. (2015). Investigación documental sobre calidad de la educación en Instituciones Educativas del contexto iberoamericano. En Revista Entramados- Educación y Sociedad, Año 2, No. 2, septiembre 2015 Pp. 107- 124. Recuperado en https://dialnet.unirioja.es/descarga/ articulo/5236201.pdf

Boni, A., Gasper, D. (2011). La Universidad como debiera ser. Propuestas desde el desarrollo humano para repensar la calidad de la Universidad. Editorial Sistema. Biblioteca Hegoa. Universidad del País Vasco. España. Pag 93 - 109. Recuperado de http://biblioteca.hegoa.ehu.es/system/ebooks/18317/original/La_universidad_ como_debiera_ser.pdf?1300270935

Booth T.y Ainscow M. (2000). Guía para la evaluación y mejora de la educación inclusiva. Consorcio Universitario Para la Educación Inclusiva. Departamento de Psicología Evolutiva y de la Educación. Facultad de Formación del Profesorado 
y Educación. Universidad Autónoma de Madrid. Recuperado en http://www. ite.educacion.es/formacion/materiales/126/cd/unidad_3/material_M1/guia_ indicadores_inclusiva_unesco.pdf

Braslavsky C. (2004). Diez factores para una educación de calidad para todos en el siglo XXI. Documento Básico. XIX Semana Monográfica de la Educación. Educación de calidad para todos: Iniciativas Iberoamericanas. Fundación Santillana. Recuperado en www.oei.es/historico/.../diez_factores_educacion_calidad_siglo_xxi_braslavsky. pdf

Braslavsky C.y Cosse G. (2006). Las actuales reformas educativas en América Latina: cuatro actores, tres lógicas y ocho tensiones. REICE (Revista Electrónica Iberoamericana sobre Calidad, Eficacia y Cambio en Educación) 2006, Vol. 4, No. 2e. Recuperado de http://www.redalyc.org/pdf/551/55140202.pdf

Braslavsky C. (2006). Diez factores para una educación de calidad para todos en el siglo XXI. REICE (Revista Electrónica Iberoamericana sobre Calidad, Eficacia y Cambio en Educación) 2006, Vol. 4, No. 2e. Recuperado de http://www.rinace.net/ arts/vol4num2e/art5.pdf

Caballero, I. G. (2004). Cuaderno de Trabajo Educación para el Desarrollo. Etxea: UNESCO Recuperado de http://educaciondesarrollo.fongdcam.org/files/2012/01/ caballero.pdf

CCA, (2014) Manual de Acreditación. Costa Rica: CCA Recuperado de: http:// www.cca.ucr.ac.cr/sites/default/files/documentos/manual_de_acreditacion_4.3eariasformato.pdf

CCA. (2014). Modelo SIGCCA. Modelo de Sistema Interno de Gestión de Calidad del CCA. Costa Rica. Recuperado en www.cca.ucr.ac.cr/sites/default/files/archivos adjuntos/modelo_sigcca.docx

Contreras, L. (2011). El proceso de institucionalización de la calidad en la gestión pública en el gobierno del estado de México 1999-2009. Revista Espacios Públicos, vol. 14, núm. 31, mayo-agosto, 2011, pp. 8-31. Recuperado en http://www.redalyc. org/pdf/676/67621192002.pdf

De Miguel Díaz, M. y Apodaca U. P. (2009). Criterios para evaluar el impacto de los planes de evaluación de la calidad de la educación superior. Revista de Educación, 349. Mayo-agosto 2009, pp. 295-310. Recuperado de http://www.ehu. eus/documents/1463215/1504238/De_Miguel_Apodaca_RevEduc_2009.pdf 


\section{LA INSTITUCIONALIZACIÓN DEL SISTEMA DE ASEGURAMIENTO DE CALIDAD EN LA EDUCACIÓN SUPERIOREN HONDURAS}

Espinoza, O. D. (2010). Los sistemas de aseguramiento de la calidad en la educación superior en América Latina. Recuperado de http://www.revistaakademeia.cl/wp/wpcontent/uploads/2010/08/

Espinoza, O. y Gonzáles, L. (2012). Estado actual del Sistema de Aseguramiento de Calidad y Régimen de Acreditación de la Educación Superior en Chile. Revista de la Educación Superior. Vol. XLI (2), No. 162, abril - junio, pp. 87-109. D.F. México. Recuperado de http://www.redalyc.org/pdf/604/60425033005.pdf

González, L.E; Espinoza, O. (2008) Calidad de la educación superior: concepto y modelos. Calidad en la educación $N^{\circ} 28$, julio págs. 247-276. Recuperado de https:// coyunturapolitica.files.wordpress.com/2011/09/calidad-de-la-educacion-superiorconceptos-y-modelos.pdf

Habermas, J. (1987) The Theory of Communicative Action, Vol. II, Lifeworld and System: A Critique of Functionalist Reason, trans. T. McCarthy. Boston, MA: Beacon Press.

Lujambio, A. I. (2010). Modelo de Gestión Educativa Estratégica. Secretaría de Educación Pública. México. Recuperado en diciembre 2016 en http://edu.jalisco. gob.mx/cepse/sites/edu.jalisco.gob.mx.cepse/files/modelo_de_gestion_educativa_ estrategica_modulo_1_pec.pdf

Max-Neef, M. (2000). Desarrollo a Escala Humana. Montevideo, Uruguay: Icaria Editorial. Recuperado en https://www.max-neef.cl/descargas/Max_NeefDesarrollo_a_escala_humana.pdf

Mesa, M. (2000). La educación para el desarrollo en la Comunidad de Madrid. Tendencias y estrategias para el siglo XXI: Informe a la Dirección General de Cooperación y Voluntariado de la Comunidad de Madrid. Recuperado de http://www. ceipaz.org/images/contenido/Precedentes, $\% 20$ desarrollo\%20y\%20madur.pdf

Morín, E. (1999). Los siete saberes necesarios para la educación del futuro. UNESCO. Recuperado de http://unesdoc.unesco.org/images/0011/001177/117740so.pdf

Nussbaum, M. C. (2010). Cultivating Humanity. A classical defense of reform in liberal education. Massachusetts: Harvard University Press, 1997 y Not for Profit. Why Democracy Needs Humanities, Princeton Books, Princeton. Recuperado en https://translate.google.hn/translate?hl=es\&sl=en\&u=http://www.hup.harvard.edu/ catalog.php\%3Fisbn\%3D9780674179493\%26content\%3Dreviews\&prev=search 
Schmelkes, S.(1994). Hacia una mejor calidad de nuestras escuelas. Colección INTERAMER, ISSN 1021-4666; no. 32.OEA/OAS.

Seibold J. (2000). La calidad integral en educación. Reflexiones sobre un nuevo concepto de calidad educativa que integre valores y equidad educativa. Ediciones OEI. Revista Iberoamericana de Educación. España. Núm. 23, mayo - agosto. Recuperado en diciembre 2016 en http://rieoei.org/rie23a07.htm

Sen, A. (2000). Desarrollo y Libertad. Editorial Planeta. Buenos Aires, Argentina. Recuperado de http://www.palermo.edu/Archivos_content/2015/derecho/pobreza multidimensional/bibliografia/Sesion1_doc1.pdf

Sen, A. (2004). Nuevo examen de la desigualdad. Editorial Alianza. España. Texto completo en http://www.casadellibro.com/libro-nuevo-examen-de-la desigualdad/9788420629513/666970

Sen, A. (2009). La idea de la justicia. Harvard University Press. U.S.A. Texto completo disponible en http://www.hup.harvard.edu/catalog.php?isbn=9780674060470

Serrano, A. (2007). Educación Superior para el Desarrollo Humano Sostenible: (Una propuesta de investigación institucional en la UNAH). Modulo I, Antología VI Promoción Doctorado en Ciencias Sociales, Aula del Doctorado en Ciencias Sociales. Recuperado de www.tzibalnaah.unah.edu.hn/bitstream/handle/123456789/.../T$\mathrm{PhD} \% 2000034 . p d f$

Tolbert, P., Zucker, L. (1996). The Institutionalization of Institutional Theory. ILR Collection. Pag. 175-190. Cornell University ILR School Recuperado en octubre de 2016 en http://digitalcommons.ilr.cornell.edu/cgi/viewcontent.cgi?article=1430\&co ntext $=$ articles

Tomasevski, K. Los derechos económicos, sociales y culturales el derecho a la educación. Recuperado de http://revistas.uptc.edu.co/revistas/index.php/memorias local/article/view/996

Tünnermann B., C. (2008). La calidad de la educación superior y su acreditación: la experiencia centroamericana. Revista SciELO. Brasil. Vol. 13, $\mathrm{N}^{\circ}$ 2, junio. Recuperado de http://www.scielo.br/scielo.php?script=sci_arttext\&pid $=$ S1414-40772008000200005

Tünnermann B., C. (2011). La educación superior frente a los desafíos contemporáneos. Universidad Centroamericana. Managua, Nicaragua. Recuperado de http://www. 


\section{LA INSTITUCIONALIZACIÓN DEL SISTEMA DE ASEGURAMIENTO DE CALIDAD EN LA EDUCACIÓN SUPERIOREN HONDURAS}

unl.edu.ar/iberoextension/dvd/archivos/panelistas/ponencia_carlos_tunnermann berheim.pdf

UNESCO. (2009i). Conferencia Mundial sobre la Educación Superior. Recuperado el marzo de 2015 de http://www.me.gov.ar/spu/documentos/Declaracion_conferencia Mundial_de_Educacion_Superior_2009.pdf

UNESCO (2009ii). La Educación: un derecho humano. Recuperado en octubre de 2016 en http://unesdoc.unesco.org/images/0018/001849/184967s.pdf

UNESCO (2015) Declaración de Incheon, Educación 2030: Hacia una educación inclusiva y equitativa de calidad y un aprendizaje a lo largo de la vida para todos Recuperado de http://www.unesco.org/new/fileadmin/MULTIMEDIA/FIELD/ Santiago/pdf/ESP-Marco-de-Accion-E2030-aprobado.pdf

Yarzábal, L. (2002). Factores de calidad para las Universidades Latinoamericanas. Ponencia preparada para la sesión concurrente sobre calidad de la educación superior. Taller de Facultad. Sistema Universitario de Ana G. Méndez. Puerto Rico. Recuperado de https://www.academia.edu/3048612/Factores_de_calidad_de_la_ educaci $\% \mathrm{C} 3 \% \mathrm{~B} 3 \mathrm{n} \_$superi 